\title{
The Treatment of Tacrolimus (FK506) for Psoriasis: One Case Report and Literature Analysis
}

\author{
Wei-gang $\mathrm{Cao}^{1}$ and Bao-an Qiu ${ }^{2}$
}

Psoriasis is a chronic recurrent inflammatory dermatosis, which is characterized by epidermal proliferation and erythema scales. Its etiology and pathogenesis are still unknown and treatment is difficult. The concentration of tacrolimus for the treatment of psoriasis has not been reported at home and abroad. In this report, we detected the tacrolimus plasma concentration and hope to provide a certain reference value for the clinical treatment of psoriasis.

Key words: Tacrolimus; Psoriasis; Treatment

$\mathrm{P}$ soriasis is a chronic recurrent inflammatory dermatosis, which is characterized by epidermal proliferation and erythema scales. Its etiology and pathogenesis are still unknown and treatment is difficult. Reports on the use of $0.1 \%$ and $0.03 \%$ tacrolimus ointment to treat psoriasis can be retrieved, but oral tacrolimus systemic treatment has not been reported in our country, the concentration of tacrolimus for the treatment of psoriasis has not been reported at home and abroad. In this report, a patient treated with oral tacrolimus for psoriasis had received good therapeutic effect, we detected the tacrolimus plasma concentration and hope to provide a certain reference value for the clinical treatment of psoriasis.

\section{CASE REPORT}

\section{General information and examination}

A 36-year-old male patient with chronic hepatitis B cirrhosis (decompensation) and psoriasis vulgaris was admitted to PLA Navy General Hospital on February 27, 2009. Two years ago, the patient was diagnosed as psoriasis vulgaris for systemic diffuse rash with itching, symptoms gradually became aggravated, he was given immune regulator, anti-inflammatory, Chinese medicine, glucocorticoid and other treatment, the symptoms disappeared, but finally it was recurrent. Physical examination revealed chronic

\footnotetext{
${ }^{1}$ Department of General Surgery, Yantai Affiliated Hospital of Binzhou Medical College, Yantai, Shandong Province 264100, China

${ }^{2}$ Department of Hepatobiliary Surgery, PLA Navy General Hospital, Beijing 100048, China

Correspondce: Bao-an Qiu, E-mail: luckqiu@medmail.com.cn
}

liver disease face, the head, face, trunk and limbs were widely distributed with miliary to soybean size red pimples, it was more obvious on the trunk, with partially confluent lesions, a clear border around the visible flush, rough surface, covered with the silverwhite dander, petechial hemorrhage was visible when the dander was scraped.

\section{Treatment and prognosis}

After the diagnosis of chronic hepatitis B cirrhosis (decompensation), treatment of orthotopic piggyback liver transplantation in general anesthesia was given on June 26, 2009, the surgery went well. Three mg FK506 was orally taken per day for the prevention of immune rejection from June 28, 2009. During postoperative recovery period, systemic rash gradually subsided, leaving only a small amount of pigmentation. The skin itch was basically eased and was disappeared completely on July18, 2009. The patient was discharged from hospital. Up to now, there's no recurrence. The treatment was listed in Table 1.

\section{DISCUSSION}

As a dermatopathic common disease with a prevalence rate of about $1 \%$ to $3 \%,{ }^{1}$ psoriasis is characterized by $\mathrm{CD} 4^{+} \mathrm{T}$ lymphocyte-mediated autoimmune disorder, ${ }^{2}$ inflammatory cell infiltration and keratinocyte hyperplasia. ${ }^{3}$ At present, the main treatment for psoriasis is systemic medication, such as taking retinoid drugs or immunosuppressive agents, which has an exact effect but the side effects are 
Table1. The treatment and efficacy

\begin{tabular}{|c|c|c|c|}
\hline Date & Tacrolimus dose (mg/d) & $\begin{array}{l}\text { Tacrolimus concentration } \\
(\mathrm{ng} / \mathrm{ml})\end{array}$ & $\begin{array}{c}\text { Psoriasis clinical } \\
\text { manifestations }\end{array}$ \\
\hline June 28, 2009 - June 30th, $2009^{a}$ & 3 & & +++ \\
\hline - July 7th, $2009^{\text {b }}$ & 4 & 8.6 & + \\
\hline - July 21st, 2009 & 4 & 13.6 & ++ \\
\hline - Aug. 1st, 2009 & \# & 5.3 & ++ \\
\hline - Aug. 12th, 2009 & 4 & 10.4 & + \\
\hline - Aug. 27th, $2009 *$ & 1 & 6.1 & + \\
\hline - Oct. 17th, 2009* & 3 & 8.4 & + \\
\hline - Feb. 21st, 2010** & 1 & 5.4 & + \\
\hline - Feb. 28th, 2010** & 2 & 6.4 & + \\
\hline - July 16th, 2010 & 3 & 4.8 & + \\
\hline - Aug. 16th, 2010 & 6 & 10.9 & + \\
\hline - Oct. 13th, 2010 & 1 & 4.3 & + \\
\hline Oct.13th, 2010 - So far & $1-3$ & 4.5-7.3 & + \\
\hline
\end{tabular}

Notes:

a methylprednisolone $200 \mathrm{mg}$.

b $20 \mathrm{mg}$ of basiliximab on July 2, 2009 .

* prednisolone $20 \mathrm{mg}$ per day taken orally.

** prednisolone $5 \mathrm{mg}$ per day taken orally .

\# stop oral administration of FK506 because of graft versus host disease (GVHD).

+++ skin rashes with skin itching.

++ skin rashes without skin itching.

+ little skin rashes and no itchy skin.

Space stands for no detection.

severe, and when the administration of drugs stopped it is easy to relapse. Blocking the different key targets of immune response, biologic agents have been used in the treatment of psoriasis and other immunemediated inflammatory disease in the United States and Europe. ${ }^{4}$

Tacrolimus (FK506) is a new macrocyclic lactone structure immunosuppressant isolated in 1984 by Japan's Fujisawa Pharmaceutical Company, trade name is tacrolimus. ${ }^{5}$ The action mechanism of FK506 -FKBP-complex (FK506 combining with FK506 binding protein in the $\mathrm{T}$ lymphocytes cytoplasm) in vivo are inhibition of phosphorylase phosphatase activity of calmodulin, the anti-host reaction and delayed hypersensitivity. ${ }^{5}$ Mrowietz et al in 1996 firstly reported a double-blind, placebo-controlled, multi-center clinical trials that 50 cases of severe refractory plaque psoriasis patients who were given oral administration of tacrolimus $0.05 \mathrm{mg} / \mathrm{kg}$ per day, compared with placebo group, there was no significant difference on the lesions area improvement after three weeks, then the dose of Tacrolimus was increased to $0.1 \mathrm{mg} / \mathrm{kg}$ per day, clinical symptoms improved three weeks later. Then the tacrolimus dose was increased to $0.15 \mathrm{mg} / \mathrm{kg}$ per day until the nine weeks comprehensive treatment. ${ }^{6}$ It has shown that the regional lesions was decreased by $83 \%$ in treatment group and by $47 \%$ in the placebo group, tacrolimus is an effective method for treatment of refractory severe psoriasis, which was well tolerated and has minor side effects. Its adverse reactions were diarrhea, abdominal pain, nausea, paresthesia, tremor, elevated blood pressure, heart palpitations, it also has some impacts on the function of kidney and liver. The side effects of FK506 is closely related to plasma concentration, its treatment concentration was 5-20 $\mathrm{ng} / \mathrm{ml}$, thus it's necessary to strengthen the monitoring of plasma concentration and formulate a reasonable individual and secure treatment scheme. ${ }^{7}$

The reports of $0.1 \%$ and $0.03 \%$ tacrolimus ointment treatment for psoriasis can be retrieved, but oral FK506 systemic treatment of psoriasis has not been reported at home. Mrowietz et al reported the dose and duration of FK506 treatment for refractory psoriasis, but exact plasma concentration of FK506 in the treatment of psoriasis has not been reported. 
It is believed that oral administration of FK506 has a good effect for the treatment of psoriasis. During the treatment, we had detected the FK506 blood drug concentration, provided some reference values for clinical treatment of psoriasis, but the best FK506 concentration for treatment of psoriasis is still need to be clarified.

\section{REFERENCES}

1. Greaves MW, Weinstein GD. Treatment of psoriasis. N Engl J Med 1995; 332(9):581-589.

2. Griffiths CE. The immunological basis of psoriasis. J Eur Acad Dermatol Venereol 2003; 17(Suppl 2) :1-5.

3. Li X, Fan X, Zhang K, Yin G, Liu Y. Influence of psoriatic peripheral blood $\mathrm{CD}^{+} \mathrm{T}$ and $\mathrm{CD}^{+} \mathrm{T}$ lymphocytes on C-myc,
Bcl-xL and Ki67 gene expression in keratinocytes. Eur J Dermatol 2007; 17(5): 392-396.

4. Sfikakis PP. The first decade of biologic TNF antagonists in clinical practice: lessons learned, unresolved issues and future directions. Curr Dir Autoimmun 2010; 11:180-210.

5. Rauch MC, San Martín A, Ojeda D, Quezada C, Salas M, Cárcamo JG, et al. Tacrolimus causes a blockage of protein secretion which reinforces its immunosuppressive activity and also explains some of its toxic side - effects. Transpl Immunol 2009; 22(1-2):72-81.

6. Europen FK506 Multicenter Psoriasis Study Group. Systemic tacrolimus (FK506) is effective for treatment of psoriasis in a double-blind, placebo-controlled study. Arch Dermatol 1996; 132:419-423.

7. Karaalp A, Demir D, Goren MZ, Akc A, ISkender E, Yananl $\mathrm{HR}$, et al. Therapeutic drug monitoring of immunosuppressant drugs in Marmara University Hospital. Ther Drug Monit 2004; 26: 263-266. 